\title{
Injection Mismatch \\ for the SNS Accumulator Ring
}

BNL/SNS TECHNICAL NOTE

NO. 080

J. Beebe-Wang, C. R. Prior

June 1, 2000

COLLIDER-ACCELERATOR DEPARTMENT BROOKHAVEN NATIONAL LABORATORY UPTON, NEW YORK 11973 


\title{
Injection Mismatch for the SNS Accumulator Ring
}

\author{
Joanne Beebe-Wang \\ Brookhaven National Laboratory, Upton, NY 11973, USA \\ Christopher R. Prior \\ Rutherford Appleton Laboratory, Chilton, Didcot Oxon OX11 0QX, UK
}

\section{Introduction}

In modern accelerator/accumulator ring design, it has been a primary concern to match all the physical parameters of the linac and ring at the injection point. In general, a mismatched injection could result in an undesirable transverse emittance growth. However, Twiss parameter mismatch can be used as a tool to reduce the foil or septumhitting rate, and therefore reduce the beam loss. In this note, we first give preferred conditions for injection mismatch, which apply to a standard multi-turn injection with a foil or a septum, then demonstrate its effectiveness on the SNS accumulator ring [1].

\section{Preferred Conditions}

Mismatched injection should satisfy two preferred conditions in order to efficiently stack injected turns in phase space.

If the painting in $\left(x, x^{\prime}\right)$ phase space is controlled independently from the $\left(y, y^{\prime}\right)$ painting, the 4-D problem is reduced to 2-D. So, here we derive the first condition in $(x$, $\left.x^{\prime}\right)$ phase space. Consider an injected beam at the injection point with centroid located at $\left(X_{C}, X_{C}^{\prime}\right)$, and suppose the closed orbit is located at $\left(X_{O}, X_{O}^{\prime}\right)$. At the observation point, the particles in the injected beam have coordinates satisfying

$$
\beta_{i}\left(x^{\prime}-X_{C}^{\prime}\right)^{2}+2 \alpha_{i}\left(x-X_{C}\right)\left(x^{\prime}-X_{C}^{\prime}\right)+\gamma_{i}\left(x-X_{C}\right)^{2} \leq \varepsilon_{i},
$$

where $\alpha_{i}, \beta i$ and $\gamma_{i}$ are the values of Twiss function in the transfer line at the injection point. These particles will under go betatron oscillation around the closed orbit in subsequent revolutions about the ring satisfying 


$$
\beta_{m}\left(x^{\prime}-X^{\prime}{ }_{o}\right)^{2}+2 \alpha_{m}\left(x-X_{O}\right)\left(x^{\prime}-X^{\prime}{ }_{o}\right)+\gamma_{m}\left(x-X_{o}\right)^{2} \leq \varepsilon_{m},
$$

where $\alpha_{m}, \beta_{m}$ and $\gamma_{m}$ are the values of Twiss function in the ring at the injection point.

In terms of normalized variables

$$
\begin{aligned}
& \xi=\frac{x}{\sqrt{\beta_{x}}}, \\
& \xi^{\prime}=\sqrt{\beta_{x}} x^{\prime}+\frac{\alpha_{x}}{\sqrt{\beta_{x}}} x
\end{aligned}
$$

the closed orbit in the ring maps to

$$
\begin{aligned}
& \xi_{O}=\frac{X_{O}}{\sqrt{\beta_{m}}} \\
& \xi_{O}^{\prime}=\sqrt{\beta_{m}} X^{\prime}{ }_{O}+\frac{\alpha_{m}}{\sqrt{\beta_{m}}} X_{O}
\end{aligned}
$$

and the centroid of the injected beam maps to

$$
\begin{aligned}
& \xi_{C}=\frac{X_{C}}{\sqrt{\beta_{i}}}, \\
& \xi_{C}^{\prime}=\sqrt{\beta_{i}} X^{\prime}{ }_{C}+\frac{\alpha_{i}}{\sqrt{\beta_{i}}} X_{C} .
\end{aligned}
$$

The expression in the ring phase space simplifies to:

$$
\left(\xi-\xi_{o}\right)^{2}+\left(\xi^{\prime}-\xi_{o}^{\prime}\right)^{2} \leq \varepsilon_{m}
$$

and the expression for the injected beam becomes:

$$
\frac{\beta_{i}}{\beta_{m}}\left(\xi^{\prime}-\xi_{C}^{\prime}\right)^{2}+2 \beta_{i}\left(\frac{\alpha_{i}}{\beta_{i}}-\frac{\alpha_{m}}{\beta_{m}}\right)\left(\xi^{\prime}-\xi_{C}^{\prime}\right)\left(\xi-\xi_{C}\right)+\left(\alpha_{m}{ }^{2} \frac{\beta_{i}}{\beta_{m}}-2 \alpha_{m} \alpha_{i}+\gamma_{i} \beta_{m}\right)\left(\xi-\xi_{C}^{\prime}\right)^{2} \leq \varepsilon_{i} .(10)
$$


Then, the first condition is obtained by choosing the parameters so that:

$$
\frac{\alpha_{i}}{\beta_{i}}=\frac{\alpha_{m}}{\beta_{m}}=-\frac{X_{C}^{\prime}-X_{O}^{\prime}}{X_{C}-X_{O}} .
$$

This condition ensures the injected beam optimally positioned within the desired beam emittance in the ring. As shown by the green ellipse in Fig. 1, the injected beam becomes upright in normalized phase space with $\xi_{C}^{\prime}=0$ and its expression becomes

$$
\frac{\beta_{i}}{\beta_{m}} \xi^{\prime 2}+\frac{\beta_{m}}{\beta_{i}}\left(\xi-\xi_{C}\right)^{2} \leq \varepsilon_{i} .
$$

When the first condition is not met (brown ellipse in Fig. 1) a larger foil size is needed for the mismatched injection, (the area between the two brown dashed lines compared to the area between two green solid lines). This can result in a higher foilhitting rate.

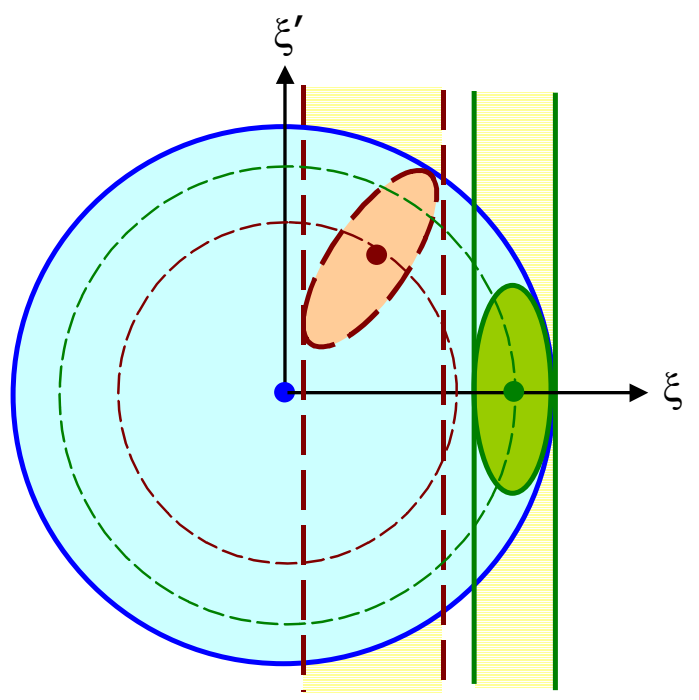

Fig. 1 Illustration of the first condition. The green and brown ellipses show injected beam meeting and failing to satisfy the first condition, respectively. The yellow areas 
between two green solid lines and between two brown dashed lines are the corresponding foil sizes in phase space required for the injection respectively.

The second condition can be derived by minimizing phase space dilution and ensures that particles with $\xi_{C}^{\prime}=0$ determine the emittance as the beam is injected. The requirement is that ellipse (12) is inside ellipse (9) and the two touch on the $\xi$-axis as illustrated by the green and blue ellipses in Fig. 2 .

With the parameterization

$$
\begin{aligned}
& \xi=\xi_{C} \sqrt{\frac{\varepsilon_{i} \beta_{i}}{\beta_{m}}} \cos \theta \\
& \xi^{\prime}=\sqrt{\frac{\varepsilon_{i} \beta_{m}}{\beta_{i}}} \sin \theta,
\end{aligned}
$$

for particles on the boundary of (12), the corresponding machine emittance is:

$$
\varepsilon_{m}(\theta)=\left(\xi_{C}+\sqrt{\frac{\varepsilon_{i} \beta_{i}}{\beta_{m}}} \cos \theta\right)^{2}+\left(\sqrt{\frac{\varepsilon_{i} \beta_{m}}{\beta_{i}}} \sin \theta\right)^{2} .
$$

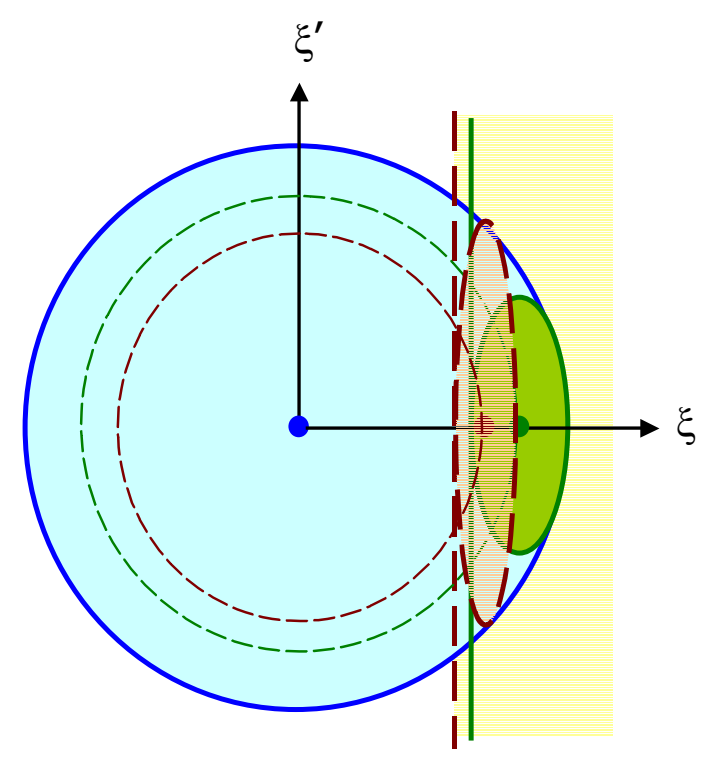

Fig. 2 Illustration of the second condition. The emittance of the circulating beam is best determined by the green ellipse, not by the brown ellipse. 
Therefore

$$
\varepsilon_{m}(0)=\left(\xi_{C}+\sqrt{\frac{\varepsilon_{i} \beta_{i}}{\beta_{m}}}\right)^{2}
$$

and

$$
\varepsilon_{m}(\theta)-\varepsilon_{m}(0)=(1-\cos \theta)\left[\varepsilon_{i}\left(\frac{\beta_{m}}{\beta_{i}}-\frac{\beta_{i}}{\beta_{m}}\right)(1+\cos \theta)-2 \xi_{C} \sqrt{\frac{\varepsilon_{i} \beta_{i}}{\beta_{m}}}\right]
$$

If $\beta_{m} \leq \beta_{i}$, assuming $\xi_{C} \geq 0,(17)$ is trivially negative. If $\beta_{m}>\beta_{i}$, the condition that the maximum emittance corresponds to $\theta=0$ gives:

$$
\varepsilon_{i}\left(\frac{\beta_{m}}{\beta_{i}}-\frac{\beta_{i}}{\beta_{m}}\right)-\xi_{C} \sqrt{\frac{\varepsilon_{i} \beta_{i}}{\beta_{m}}} \leq 0
$$

Combining (16), an alternative and more useful formulation of (18) is:

$$
\frac{\beta_{i}}{\beta_{m}} \geq\left(\frac{\varepsilon_{i}}{\varepsilon_{m}}\right)^{1 / 3}
$$

This condition ensures the injected beam has an aspect ratio corresponding to the green ellipse in Fig. 2. The emittance of the circulating beam should be determined by the green ellipse, not by the brown ellipse. Then, the foil size is determined by the green solid line, not by the brown dashed line. When this condition is not met (brown ellipse in Fig. 2), the top and bottom points of the brown ellipse determine the emittance of circulating beam. In this case, the dashed brown line determines the foil size, which may yields a higher foil-hitting rate.

\section{Simulation Results}

Computer simulations were performed in order to study the foil-hitting properties owing to injection mismatch. All the physical quantities used in the simulations are chosen to be as close as possible to the specifications in the present design [2]. The lattice functions and other salient parameters are listed in Table 1. There are two basic painting methods - correlated and anti-correlated painting [3] - incorporated in the SNS accumulator ring design. The choice of painting schemes and the detailed bump motion 
as a function of time play important roles in the foil hitting properties. The simulation study is performed with optimized injection bumps for both correlated and anti-correlated painting schemes. Higher order effects due to space charge, magnet field error, misalignment, magnetic fringe fields are not included in this study.

In the design for the SNS injection, the accumulator ring fill-time is $1 \mathrm{msec}$. We simulate one injection/painting period (from the beginning of injection to extraction). The injected beam is assumed to have a Gaussian distribution. The foil size is chosen to be $6 \sigma_{i n j}$, regardless of injected beam size, which yields $0.3 \%$ foil missing rate. Foil hitting rate is defined by (total foil hits) / (total number of particles at the end of injection). Since each $\mathrm{H}^{+}$is expected to strip into one proton and two electrons, the first passage of the particles through injection foil is counted as an "effective initial passage" of 3. In order to calculate the current density on the foil during the injection, the foil is divided into small areas of $1.0 \mathrm{~mm}(\mathrm{H}) \times 1.0 \mathrm{~mm}(\mathrm{~V})$. The accumulation of the "effective initial passage" and subsequent passages in each small area during the $1 \mathrm{msec}$ fill-time is calculated. This calculation gives the current density in units of hits $/ \mathrm{mm}^{2} / \mathrm{msec}$. Then the maximum temperature on the foil is calculated from the maximum current density with a simulation code, which includes the foil heating during the filling and the foil cooling between the fill-times. It was found that, in most cases, the location of the injected beam center is the hottest spot on the foil due to the Gaussian distribution of SNS injected beam and the "effective initial passage" of 3 .

Table 1 Design parameters used in the simulation study.

\begin{tabular}{|l|c|}
\hline Beam Kinetic Energy & $1 \mathrm{GeV}$ \\
\hline Beam Average Power & $2.0 \mathrm{MW}$ \\
\hline Protons per Pulse & $2.08 \times 10^{14}$ \\
\hline Pulse Repetition Rate & $60 \mathrm{~Hz}$ \\
\hline Proton Revolution Period & $945 \mathrm{nsec}$ \\
\hline Ring Circumference & $248 \mathrm{~m}$ \\
\hline Number of Turns Injected & 1060 \\
\hline Ring Fill Time & $1.0 \mathrm{msec}$ \\
\hline Tunes $v_{x} / v_{y}$ & $6.3 / 5.8$ \\
\hline$\beta_{x}$ and $\beta_{y}$ at Injection & $8.98 / 13.26 \mathrm{~m}$ \\
\hline$\alpha_{x}$ and $\alpha_{y}$ at Injection & $0.068 / 0.046$ \\
\hline Dispersion at Injection & $0.0 \mathrm{~m}$ \\
\hline Un-normalized Emittance $(99 \%)$ & $160 \pi \mathrm{mm}-\mathrm{mr}$ \\
\hline
\end{tabular}


In the current SNS linac design, the normalized RMS emittance of the injected beam is $0.5 \pi \mathrm{mm}$-mrad. In order to investigate the impact of larger linac emittances during future operations, the range $0.5-3.0 \pi \mathrm{mm}-\mathrm{mrad}$ has been studied. The maximum mismatch in the $\beta$-functions for a given $\varepsilon_{i}$ is determined by exactly meeting the second injection condition (13). In our investigation, since the required full normalized emittance is $\varepsilon_{m, M A X}=289 \pi \mathrm{mm}-\mathrm{mrad}$, the optimum range is $\left[\beta_{i} / \beta_{m}\right]_{\min }=0.12-0.22$. The studies are performed with condition $\beta_{i} / \beta_{m}=0.22-1.0$ in order to ensure that all the cases presented satisfy two preferred conditions.

The foil hitting rate and maximum foil temperature are investigated for one matching condition and three different degrees of mismatch. Fig. 3 illustrates the phase space in these four cases. The large blue circle represents the circulating beam in the ring. The red circle and red vertical line represent the injected beam and the corresponding foil edge with matched Twiss parameters. The green, blue and magenta ellipses and lines represent three mismatched cases with $\beta_{i} / \beta_{m}=0.64,0.36$ and 0.22 , respectively (not to scale).

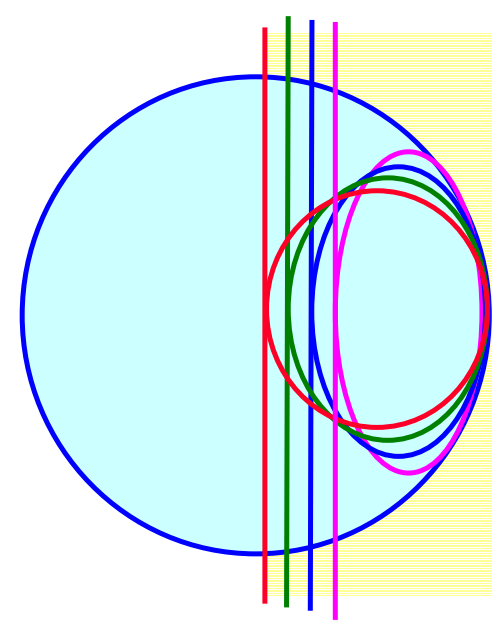

Fig. 3 Phase space illustration. The large blue circle represents the circulation beam in the ring. The red circle and red vertical line represent the injected beam and the corresponding foil edge with matched Twiss parameters. The green, blue and magenta ellipses and lines represent three mismatched cases with $\beta_{i} / \beta_{m}=0.64,0.36$ and 0.22 , respectively (not to scale).

Fig. 4(a) and Fig. 5(a) show simulation results of foil-hitting rate vs. emittance of the injected beam for the four cases with correlated and anti-correlated paintings respectively. The color code in Fig. 4 and Fig. 5 is consistent with Fig. 3. It is easy to see 
that, when both preferred conditions are satisfied, the higher degree of mismatch corresponds to a lower foil-hitting rate, because the smaller beam size due to a smaller $\beta$ function results in a smaller required foil size. Therefore, in order to reduce the beam loss at the injection, it is advantageous to have higher degree of mismatch.

However, a higher degree of mismatch is also associated with higher current density on the foil owing to the smaller injected beam size, and this yields higher foil temperatures. Fig. 4(b) and Fig. 5(b) show the maximum foil temperature vs. the emittance of injected beam for the four cases with correlated and anti-correlated paintings respectively. The maximum foil temperatures are obtained from the current densities on the foil by using the relationship shown in Fig. 6, which is extracted from the results of simulations [4] with the designed SNS parameters [2]. These simulation results clearly demonstrate how foil temperature depends on the injected beam emittance and the degree of mismatch - a higher degree of mismatch and smaller injected beam emittance yields a higher foil temperature. If the maximum temperature during injection exceeds $2000 \mathrm{~K}$ (light blue lines in Fig. 4(b) and Fig. 5(b)), the foil lifetime would be dramatically reduced.

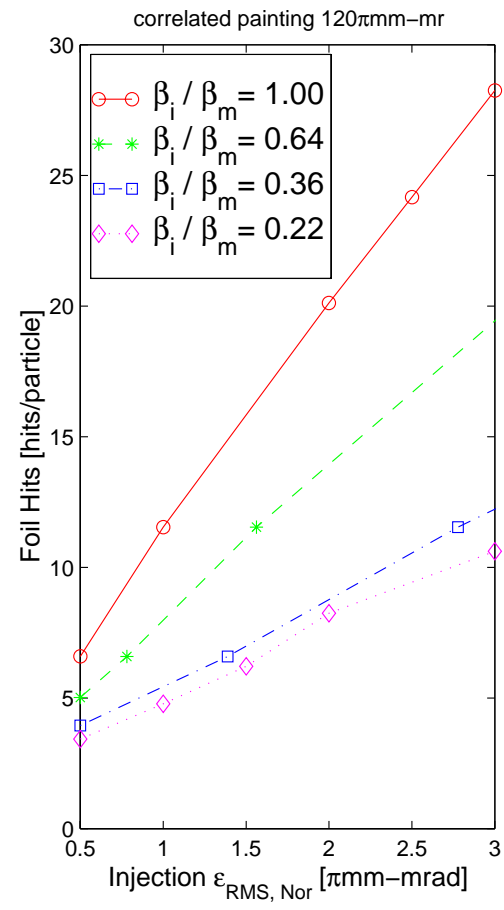

(a)

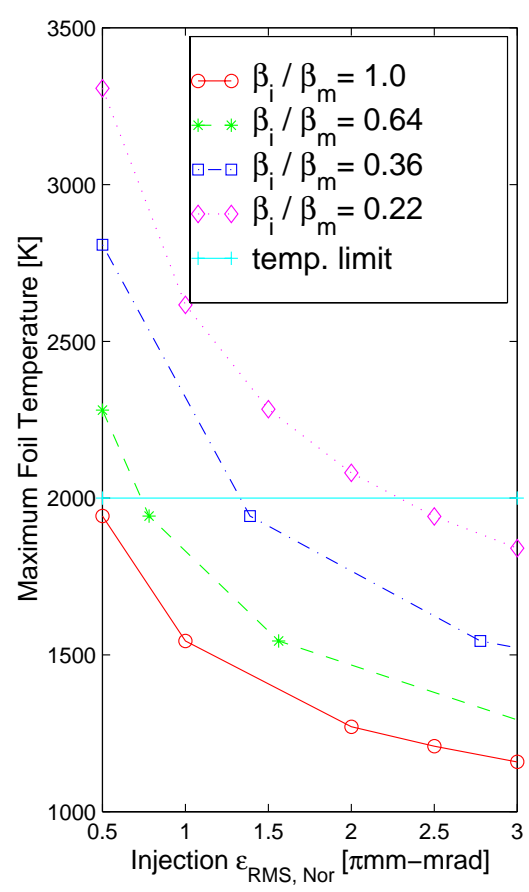

(b)

Fig.4 Foil-hitting properties due to different degrees of injection mismatch for correlated painting. (a) foil-hitting rate vs. injected beam emittance; (b) foil temperature vs. injected beam emittance. 


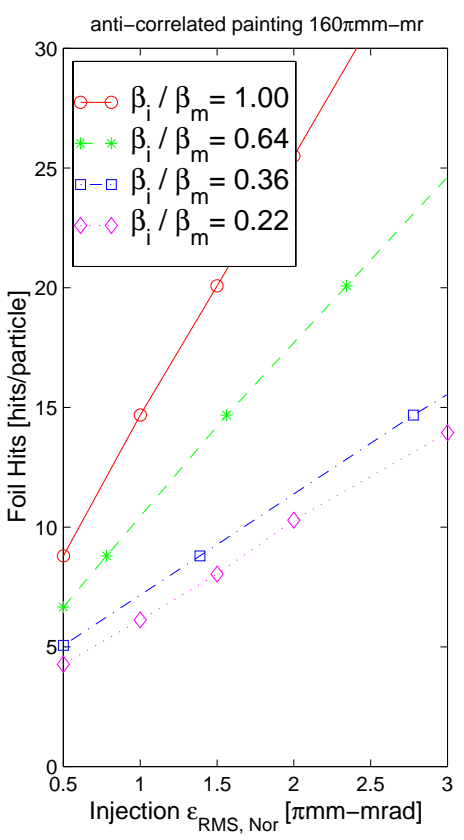

(a)

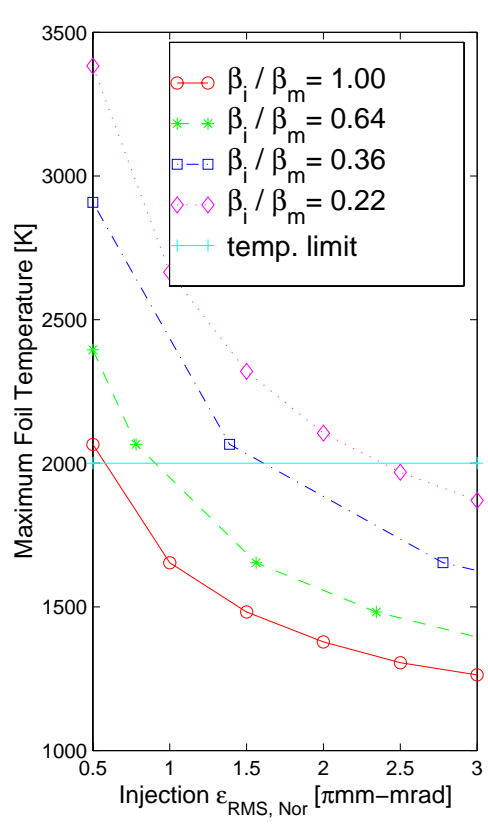

(b)

Fig.5 Foil-hitting properties due to different degrees of injection mismatch for anticorrelated painting. (a) foil-hitting rate vs. injected beam emittance; (b) foil temperature vs. injected beam emittance.

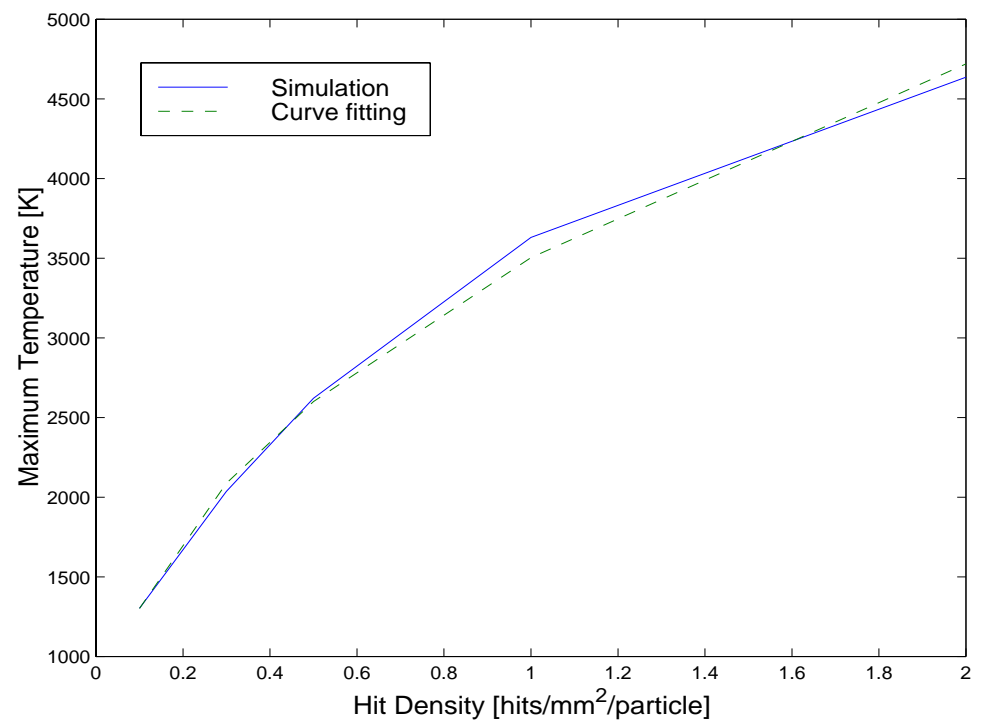

Fig. 6 The maximum foil temperature vs. current density on foil, which is extracted from the simulation results [4] with the designed SNS parameters [2]. 


\section{Injection Errors due to Mismatch}

As mentioned in the first section, injection errors due to mismatch may cause transverse emittance increase in the circulating beam. Injection errors can be categorized into three types: steering mismatch, dispersion mismatch and Twiss function mismatch. In this section, we first investigate how these mismatch errors in the SNS injection affect the transverse emittance growth. Next we show that these effects are small compared with the total emittance of the SNS beam at the end of injection painting.

\section{a) Steering mismatch}

There could be errors in phase space locations of either the centroid of the injected beam or the closed orbit of the circulating beam. If the beam at has its centroid displaced from the design values, or an incorrect closed orbit, the additional phase space displacement $\left(\Delta x, \Delta x^{\prime}\right)$ between the centroid and the closed orbit gives an error in injection painting. As a result, there is an emittance error in the circulating beam. It has been shown by D. A. Edwards and M. J. Syphers [5] that the beam size $\sigma_{M}$ becomes $\sigma$ when a steering mismatch $\left(\Delta x, \Delta x^{\prime}\right)$ is introduced:

$$
\frac{\sigma^{2}}{\sigma_{M}^{2}}=1+\frac{1}{2} \frac{\Delta x^{2}+\left(\beta \Delta x^{\prime}+\alpha \Delta x\right)^{2}}{\sigma_{M}^{2}} .
$$

For SNS injection, the steering mismatch is estimated [6] to be $\Delta x=0.2 \mathrm{~mm}$ and $\Delta \mathrm{x}^{\prime}=0.2 \mathrm{mrad}$, which yields $\Delta \sigma=0.8 \mathrm{~mm}$ or $\Delta \varepsilon_{\mathrm{m}}=0.3 \pi \mathrm{mm}-\mathrm{mrad}$ (un-normalized).

\section{b) Dispersion mismatch}

Mismatch in the dispersion function causes a mismatch of the trajectories of offmomentum particles. Its impact on the beam size is [5]:

$$
\frac{\sigma^{2}}{\sigma_{M}^{2}}=1+\frac{1}{2} \frac{\Delta D^{2}+\left(\beta \Delta D^{\prime}+\alpha \Delta D\right)^{2}}{\sigma_{M}^{2}} \sigma_{p}^{2} .
$$

For SNS injection, dispersion function mismatch is estimated [6] to be $\Delta \mathrm{D}=5 \mathrm{~cm}$ and $\Delta \mathrm{D}^{\prime}=0.02$, which yields $\Delta \sigma=0.6 \mathrm{~mm}$ or $\Delta \varepsilon_{\mathrm{m}}=0.2 \pi \mathrm{mm}-\mathrm{mrad}$ (un-normalized). 


\section{c) Twiss function mismatch}

If the Twiss functions $\alpha, \beta$ and $\gamma$ are slightly different from the designed values $\alpha_{M}$, $\beta_{\mathrm{M}}$ and $\gamma_{\mathrm{M}}$, there will be a transverse emittance error in the circulating beam. Its impact on the beam size is [5]:

$$
\frac{\sigma^{2}}{\sigma_{M}^{2}}=\frac{1}{2}\left(\beta \gamma_{M}+\beta_{M} \gamma-2 \alpha \alpha_{M}\right) \text {. }
$$

For SNS injection, the Twiss function mismatch is estimated [6] to be $\Delta \alpha / \alpha_{M}=\Delta \beta / \beta_{M}=$ 0.025 , which yields $\Delta \sigma=0.02 \mathrm{~mm}$ or $\Delta \varepsilon_{m}=0.01 \pi \mathrm{mm}-\mathrm{mrad}$ (un-normalized).

Clearly, compared to the total emittance of the SNS beam at the end of injection painting, $\varepsilon_{\mathrm{m}}=160 \pi \mathrm{mm}$-mrad (un-normalized), the injection errors have minor impact on emittance growth.

\section{Discussion and Conclusions}

Twiss parameter mismatch is a useful tool to reduce the foil-hitting rate thereby reducing the beam loss due to scattering at the injection. However, the resulting smaller size of the injected beam is accompanied by higher current density on the foil, and hence a higher temperature. The mismatch for the SNS injection should be optimized to reduce the foil-hitting rate while keeping the maximum temperature under operation limit (hitherto assumed to be $2000 \mathrm{~K}$ [4]) to maintain an adequate foil lifetime.

\section{References}

[1] SNS Project Office, "Spallation Neutron Source Design Manual" (1998).

[2] SNS Project Office, "SNS Parameter List", SNS-100000000-PL0001-R02 (2000).

[3] J. Beebe-Wang et al, "Transverse Phase Space Painting For SNS Accumulator Ring Injection”, p. 1743. Proc. of PAC99 (1999).

[4] C. J. Liaw, Private communications, BNL, April 2000.

[5] D. A. Edwards and M. J. Syphers, "An introduction to the Physics of High Energy Accelerators", John Wiley \& Sons, Inc., (1993).

[6] D.Raparia, Private communications, BNL, April 2000. 